\title{
Caracterização e análise estrutural da hidroxisodalita sintetizada a partir de amostras de solo amazônico
}

\section{(Characterization and structural analysis hydroxysodalite synthesized from Amazonian soil samples)}

\author{
V.A.A. de Freitas, J. S. V. Lima, P. R. da C. Couceiro \\ Departamento de Química, Universidade Federal do Amazonas, Av. Gal. Rodrigo Octávio Jordão Ramos 3000 \\ Aleixo, Manaus, AM 69077-000 \\ victoraugusto.freitas@gmail.com
}

\begin{abstract}
Resumo
As zeólitas desde sua descoberta, na forma natural, vêm promovendo grande interesse em aplicações tecnológicas como peneiras moleculares, trocadores iônicos, catalisadores e, principalmente, adsorventes. As zeólitas sintéticas geralmente são preparadas com soluções aquosas saturadas de sais de aluminatos e silicatos. Mas, rejeito industrial do processo de beneficiamento de caulim tem sido empregado para sintetizar zeólita A e hidroxisodalita, devido a razão equimolar Si/Al. Nesse trabalho foi sintetizado a zeólita do tipo hidroxisodalita $\left[\mathrm{Na}_{6}\left(\mathrm{AlSiO}_{4}\right)_{6} \cdot 8 \mathrm{H}_{2} \mathrm{O}\right]$ a partir da fração fina de duas amostras de solo amazônico - rico em caulinita $\left[\mathrm{Al}_{2} \mathrm{Si}_{2} \mathrm{O}_{5}(\mathrm{OH})_{4}\right]$ que é basicamente composta de $\mathrm{Al}$ e $\mathrm{Si}$ - usando o método hidrotermal. A caracterização por espectrofotometria de absorção no infravermelho com transformada de Fourier, análise termogravimétrica e térmica diferencial e difração de raios X confirmaram que as amostras in natura são majoritariamente compostas por caulinita com 69 a $71 \%$ de abundância. O refinamento estrutural de Rietveld mostrou que o produto da síntese é constituída de uma fase cristalina de hidroxisodalita de estrutura cúbica (grupo espacial P $\overline{4} 3 \mathrm{n}, \# 218$ ) com parâmetros de rede $a=0,88468$ e 0,88594 nm. Os dados ajustados permitiram estimar o tamanho médio dos cristalitos $d=57 \mathrm{~nm}$ (ambas amostras) e a área superficial $S=44 \mathrm{e} 41 \mathrm{~m}^{2} \cdot \mathrm{g}^{-1}$.

Palavras-chave: síntese hidrotermal, refinamento Rietveld, caulinita, metacaulinita, hidroxisodalita.
\end{abstract}

\begin{abstract}
Zeolites since their discovery in natural form have promoted a great interest in technological applications as molecular sieves, ion exchangers, catalysts, and especially adsorbents. The synthetic zeolites are usually prepared with saturated aqueous solutions of salts of aluminates, and silicates. But the process of industrial waste processing kaolin has been used to synthesize zeolite A and hydroxysodalite due to equimolar Si/Al ratio. In this work zeolite type hydroxysodalite, $\mathrm{Na}_{6}\left[\mathrm{Si}_{6} \mathrm{Al}_{6} \mathrm{O}_{4}\right]_{6} .8 \mathrm{H}_{2} \mathrm{O}$ was synthesized from the fine fraction of two samples from Amazonian soil - rich in kaolinite, $\left[\mathrm{Al}_{2} \mathrm{Si}_{2} \mathrm{O}_{5}(\mathrm{OH})_{4}\right]$, which is basically composed of $\mathrm{Al}$ and Si, using hydrothermal method. Characterization by FT-IR, TGA/DTA and XRD confirmed that the samples in natura are mainly constituted by kaolinite with 69 and $71 \%$ abundance. Rietveld structural refinement showed that the synthesis product consists of a crystalline phase hydroxysodalite of cubic structure (space group P $\overline{4} 3 \mathrm{n}$, \#218) with lattice parameters $a=0.88468$ and $0.88594 \mathrm{~nm}$. The fitted data allowed us to estimate the average crystallite size $d=57 \mathrm{~nm}$ (both samples), and the surface area, $S=44$ and $41 \mathrm{~m}^{2} \mathrm{~g}^{-1}$ for the samples.
\end{abstract}

Keywords: hydrothermal synthesis, Rietveld refinenment, kaolinite, metakaolinite, hydroxysodalite.

\section{INTRODUÇÃO}

As zeólitas são aluminossilicatos cristalinos formados por uma rede tridimensional de tetraedros de $\mathrm{AlO}_{4}$ e $\mathrm{SiO}_{4}$ ligados entre si por átomos de oxigênio. A deficiência de carga das zeólitas - gerada pela substituição isomórfica do $\mathrm{Si}^{4+}$ pelo $\mathrm{Al}^{3+}$ - é compensada por cátions alcalinos e/ou alcalinos terrosos situados nas cavidades dos anéis de oxigênio [1], com dimensões moleculares de 0,3 a $15 \mathrm{~nm}$, que pode ser preenchido por moléculas de água ou outras [2].

As zeólitas são compostas por um grande número de minerais naturais e sintéticos que apresentam características comuns [3]. Devido às suas propriedades físicas e químicas são amplamente utilizadas como peneiras moleculares, trocadores iônicos, catalisadores e, principalmente, adsorventes [4].

As zeólitas sintéticas são preparadas a partir de soluções aquosas saturadas de sais de aluminatos e silicatos, de composição definida, sob condições de temperatura (25 a $300{ }^{\circ} \mathrm{C}$ ) e pressão pré-determinadas. Sendo assim, fazendo variar a composição da solução (ou gel de síntese) e as condições operacionais, é possível sintetizar materiais com características estruturais e composições químicas diferentes $[3,5]$.

Material de partida como rejeito industrial do processo de beneficiamento de caulim, de indústrias da região 
amazônica, tem sido empregado para sintetizar zeólita A e hidroxisodalita. Esse rejeito é composto essencialmente por caulinita (argilomineral 1:1) e possui razão $\mathrm{SiO}_{2} /$ $\mathrm{Al}_{2} \mathrm{O}_{3}$ igual à requerida para esses dois tipos de zeólitas [6]. Assim, o presente trabalho teve por objetivo sintetizar zeólita tipo hidroxisodalita a partir de amostras da fração fina de solo - rica em caulinita como fonte natural de silício e alumínio - da Formação Alter do Chão, na Amazônia Ocidental. Utilizou-se o método Rietveld de refinamento de dados de difração de raios X (DRX), método do pó, para o estudo estrutural.

\section{Hidroxisodalita}

O mineral zeolita tipo sodalita pode ser descrito pela fórmula geral $\mathrm{M}_{8}\left[\mathrm{ABO}_{4}\right]_{6} \mathrm{X}_{2}$, onde $\mathrm{M}$ é um cátion monovalente, como $\mathrm{Na}^{+}, \mathrm{Li}^{+}$e $\mathrm{Ag}^{+}$, Fig. 1 [7]. A e B são espécies capazes de formar tetraedros ( $\mathrm{Al}$ e $\mathrm{Si}$ ) e $\mathrm{X}$ pode ser uma variedade de ânions mono e divalentes. A estrutura está baseada em uma cadeia octaédrica truncada formada pela união de átomos de $\mathrm{Si} \mathrm{e} \mathrm{Al}$ ligado tridimensionalmente [8, 9].

A sodalita mais conhecida e caracterizada é aquela onde o ânion monovalente está no centro da cadeia $\beta$ e coordenado por íons sódio, formando aglomerados tetraédricos $\mathrm{M}_{4} \mathrm{X}$ na cadeia $\beta$. A sodalita natural com cloreto, cristaliza no sistema cúbico, $a_{0}=0,887 \mathrm{~nm}$, grupo espacial $\mathrm{P} \overline{4} 3 \mathrm{n}$, com uma composição de célula unitária de $\mathrm{Na}_{8}\left(\mathrm{AlSiO}_{4}\right)_{6} \mathrm{Cl}_{2}$; na qual os oito sódios são estruturalmente equivalentes e o cloreto está no centro do octaedro truncado [10]. Sodalitas que possuem ânions hidróxido para estabilizar cargas estruturais são denominadas de hidroxisodalitas, $\mathrm{Na}_{6}(\mathrm{AlSiO} 4)_{6} .8 \mathrm{H}_{2} \mathrm{O}$ [11], mas possuem a mesma estrutura da sodalita com ordenamento cadeias $\beta$ e tamanho de poro de $0,28 \mathrm{~nm}$ [12].

\section{Método de Rietveld}

Hugo Rietveld desenvolveu um método computacional para refinar as estruturas de cristal usando dados de difração de nêutron [13]. Atualmente, é uma das técnicas mais poderosas para a análise estrutural, determinação cristalina, medidas dos parâmetros de rede e a análise


Figura 1: Cadeia $\beta$ (esquerda) e estrutura tridimensional da sodalita (direita).

[Figure 1: $\beta$ chain (left) and three-dimensional structure of sodalite (right).] quantitativa de DRX, método do pó [14, 15].

O método consiste no ajuste ponto a ponto de todas as intensidades obtidas experimentalmente $\left(\mathrm{y}_{i}{ }^{\text {obs }}\right)$ com as intensidades calculadas ( $\mathrm{y}_{i}^{\text {cal }}$ ) baseada em um determinado modelo cristalino (padrão), efeitos óticos de difração, fatores instrumentais e outras características da amostra. Os parâmetros incluídos no modelo são refinados utilizando o método dos mínimos quadrados visando à minimização do resíduo $S_{y}$ (função residual) dado por:

$$
\mathrm{S}_{\mathrm{y}}=\sum_{\mathrm{i}} \mathrm{w}_{\mathrm{i}}\left(\mathrm{y}_{\mathrm{i}}{ }^{\mathrm{obs}}-\mathrm{y}_{\mathrm{i}}^{\mathrm{obs}}\right)^{2}
$$

na qual $\mathrm{y}_{\mathrm{i}}^{\text {obs }}$ e $\mathrm{y}_{\mathrm{i}}^{\text {cal }}$ são as intensidades observada (ou experimental) e calculada, respectivamente, e $w_{i}$ é o fator de peso da distribuição no $i$-ésimo passo.

Ao final do refinamento, serão fornecidos os parâmetros estruturais como: parâmetros de rede, fator de ocupação, concentração e a largura à meia altura da reflexão (sigla inglesa, $F W H M$ ). A intensidade calculada para passo $i$ no padrão de difração tem a seguinte expressão:

$$
\mathrm{y}_{\mathrm{i}}^{\text {cal }}=\mathrm{s} \sum_{\mathrm{k}} \mathrm{L}_{\mathrm{K}}\left|\mathrm{F}_{\mathrm{K}}\right|^{2} \Phi\left(2 \theta_{\mathrm{i}}-2 \theta_{\mathrm{k}}\right) \mathrm{P}_{\mathrm{k}}+\mathrm{y}_{\mathrm{i}}^{\text {bkg }}
$$

na qual $s$ é o fator de escala, $K$ representa os índices de Miller $h k l$ para cada reflexão Bragg, $L_{K}$ contém os fatores de Lorentz, polarização e multiplicidade, $F_{K}$ é o fator de estrutura para a $K$-ésima reflexão Bragg, $\Phi\left(2 \theta_{i}-2 \theta_{K}\right)$ é a função perfil da reflexão, $P_{K}$ é a função orientação preferencial e $\mathrm{y}_{\mathrm{i}}^{\mathrm{bkg}}$ é a intensidade do "background" para o $i$-ésimo passo. Os critérios quantitativos para avaliar um bom ajuste são feitos pelo monitoramento dos $R^{\prime} s$, chamados de fatores de confiança que são no total cinco fatores. Mas, os fatores estatisticamente mais significativos são o $R_{w p}$ (fator de peso padrão) e $R_{\exp }$ (fator esperado) dados pelas expressões matemáticas:

$$
\begin{aligned}
& \mathrm{R}_{\mathrm{wp}}=\sqrt{\frac{\sum_{\mathrm{i}} \mathrm{w}_{\mathrm{i}}\left(\mathrm{y}_{\mathrm{i}}^{\text {obs }}-\mathrm{y}_{\mathrm{i}}^{\text {cal }}\right)^{2}}{\sum_{\mathrm{i}} \mathrm{w}_{\mathrm{i}}\left(\mathrm{y}_{\mathrm{i}}^{\mathrm{obs}}\right)^{2}}} \\
& \mathrm{R}_{\exp }=\sqrt{\frac{\mathrm{N}-\mathrm{P}}{\sum_{\mathrm{i}} \mathrm{w}_{\mathrm{i}}\left(\mathrm{y}_{\mathrm{i}}^{\text {obs }}\right)^{2}}}
\end{aligned}
$$

na qual $N$ é o número de passos experimentais e $P$ é o número de parâmetros ajustados [16-20].

\section{MATERIAIS E MÉTODOS}

Duas amostras de solo foram coletadas de um corte de 
estrada (seção colunar) - na base, amostra A01 e no topo, amostra A14 - localizado nas proximidades da Estrada do Turismo, dentro do perímetro urbano da cidade de Manaus (capital do estado do Amazonas) de coordenadas geográficas $3^{\circ} 5$ ' 38" S e 60 '2' 9" O. Essa área faz parte do depósito sedimentar sobre a Formação Alter do Chão [21], do período Cretáceo [22], na Amazônia Ocidental. Essas amostras foram tratadas física (secagem, desagregação, peneiramento e trituração) e quimicamente (ácido clorídrico $50 \%$ a quente) para se obter a fração fina do solo com menor grau de impurezas. A fonte de metal alcalino utilizada na síntese foi o hidróxido de sódio (Merck, em pastilhas).

$\mathrm{O}$ processo de síntese ocorreu em duas etapas. $\mathrm{Na}$ primeira, a fração fina do solo tratadas previamente foi calcinada em mufla a $600{ }^{\circ} \mathrm{C}$ por $5 \mathrm{~h}$, para garantir a total conversão da caulinita a metacaulinita, como fonte de silício e alumínio. A segunda ocorreu em condições hidrotérmicas, com a mistura de quantidades estequiometricamente aproximadas da fração calcinada e solução de hidróxido de sódio $5 \mathrm{molL}^{-1}$, seguida de aquecimento estático em mufla a $110^{\circ} \mathrm{C}$ por $24 \mathrm{~h}$. A mistura reacional utilizada na síntese teve composição molar aproximada de $1,26 \mathrm{Na}_{2} \mathrm{O}_{2} \mathrm{Al}_{2} \mathrm{O}_{3} \cdot 2 \mathrm{SiO}_{2}$. $\mathrm{nH}_{2} \mathrm{O}$. Posteriormente, cada produto obtido foi lavado e filtrado com água destilada até o $\mathrm{pH} 7$, e em seguida seco em estufa a $100{ }^{\circ} \mathrm{C}$ por $6 \mathrm{~h}$, procedimento adaptado de Maia e colaboradores [6] e Murat e colaboradores [23].

Os materiais precursor (amostras in natura e tratadas) e sintetizado foram caracterizados por diferentes técnicas tais como espectroscopia de emissão atômica em plasma indutivamente acoplado (ICP-AES), termoanálises (TG/ DTA), espectroscopia de infravermelho com transformada de Fourier (IV-TF) e difração de raios X (DRX). A fração fina de cada amostra in natura foi submetida à fusão alcalina $\left(\mathrm{Na}_{2} \mathrm{CO}_{3} / \mathrm{K}_{2} \mathrm{CO}_{3}\right)$ e dissolução por ataques ácidos $(\mathrm{HCl}$, $\mathrm{HNO}_{3}+\mathrm{H}_{2} \mathrm{SO}_{4}: \mathrm{HF}$ ) para quantificar os teores dos óxidos: $\mathrm{Al}, \mathrm{Ca}, \mathrm{Fe}, \mathrm{K}, \mathrm{Mg}, \mathrm{Na}$, Si e Ti. A solução foi analisada em um equipamento ICP-AES, modelo Spectroflame. E a perda ao fogo (PAF) foi determinada pelo método gravimétrico. Os termogramas e curvas de DTA da fração fina de cada amostra in natura foram obtidos em um termoanalisador TGA/DTA da Shimadzu, DTG-60H, com taxa de aquecimento de $10{ }^{\circ} \mathrm{C} \mathrm{min}^{-1}$, na faixa de temperatura de 25 a $900{ }^{\circ} \mathrm{C}$, sob fluxo de $\mathrm{N}_{2}$ de $50 \mathrm{mLmin}^{-1}$. A alumina como material de referência.

Os espectros de absorção na região do infravermelho foram obtidos em um equipamento FT-IR Perkin Elmer Spectrum 2000, na região entre 4.000 a $400 \mathrm{~cm}^{-1}$ com resolução de $2 \mathrm{~cm}^{-1}$, sendo as pastilhas produzidas com mistura de amostra e $\mathrm{KBr}$, na proporção 1:200 (m/m).

Os difratogramas foram obtidos em um difratômetro da Shimadzu, XRD 6000, equipado com um monocromador de grafite e tubo de raios $\mathrm{X}$ cerâmico de anodo de $\mathrm{Cu}$ (radiação da linha $K \alpha_{1}$ e $\mathrm{K} \alpha_{2}$ de comprimentos de onda, $\lambda=0,154056$ e $0,154439 \mathrm{~nm}$, respectivamente), sob corrente de $30 \mathrm{~mA} \mathrm{e}$ tensão de $45 \mathrm{kV}$, com varredura de 5 a $65^{\circ} 2 \theta$. Para efeito de calibração o Si foi usado como padrão interno. Os dados de DRX foram refinados pelo método Rietveld utilizando o programa computacional Fullprof [24, 25]. E para o refinamento estrutural da hidroxisodalita foram utilizados os parâmetros de difração padrão obtidos da base de dados da Structure Commission of the International Zeolite Association (IZA-SC) [26].

\section{RESULTADOS E DISCUSSÃO}

Após os tratamentos físicos, as frações finas das amostras A01 e A14 apresentavam visualmente coloração vermelha e amarela respectivamente, típico de ocorrência de hematita $\left(\mathrm{Fe}_{2} \mathrm{O}_{3}\right)$ e goethita $(\alpha-\mathrm{FeOOH})$, óxidos de ferro muito comuns nos solos amazônicos, considerados como impurezas nas amostras para a finalidade proposta. Essa observação está em consonância com a composição química mostrada na Tabela $\mathrm{I}$, em que os teores de $\mathrm{Fe}_{2} \mathrm{O}_{3}$ são bastantes expressivos comparados com $\mathrm{TiO}_{2}$ e soma dos teores de óxidos básicos $\left(\mathrm{\Sigma b}=\mathrm{CaO}+\mathrm{MgO}+\mathrm{K}_{2} \mathrm{O}+\mathrm{Na}_{2} \mathrm{O}\right)$.

Dessa forma, as amostras foram tratadas com solução de ácido clorídrico $50 \%$ a quente, com a finalidade de remover essas impurezas, em que teve como produto amostras, virtualmente isenta de impurezas, de cores mais claras, cinza e branco, para A01 e A14, respectivamente.

$\mathrm{A}$ razão molar $\mathrm{Si} / \mathrm{Al}$ estimada para a amostra $\mathrm{A} 01$ foi de aproximadamente $50 \%$ maior que a comparada com a amostra A14, o que sugere um teor aproximado de $15 \%$ de $\mathrm{SiO}_{2}$ (quartzo), enquanto que a amostra A14 apresentou uma razão molar $\mathrm{Si} / \mathrm{Al}$ próxima de 1 , e necessária para a síntese da hidroxisodalita.

As curvas de TGA e DTA para ambas as amostras apresentaram perfis gráficos semelhantes, conforme mostrado para a amostra A14 na Fig. 2. As curvas de TGA registradas entre 25 e $900{ }^{\circ} \mathrm{C}$ mostraram valores de perdas

Tabela I - Composição química (\%) dos principais óxidos, com nível de confiança de 95\%, e razão molar de Si e Al.

[Table I - Chemical composition (\%)of the main oxides, with a confidence level of 95\%, and molar ratio of Si and Al.]

\begin{tabular}{|c|c|c|c|c|c|c|c|}
\hline Amostra & $\mathrm{Al}_{2} \mathrm{O}_{3}$ & $\mathrm{SiO}_{2}$ & $\mathrm{Fe}_{2} \mathrm{O}_{3}$ & $\mathrm{TiO}_{2}$ & $\Sigma \mathrm{b}$ & $\mathrm{PAF}$ & $n_{2} / n_{1}$ \\
\hline A01 & 28,5 & 48,1 & 8,6 & 2,5 & 0,19 & 13,4 & 1,38 \\
\hline A14 & 34,4 & 39,6 & 6,3 & 1,7 & 0,19 & 15,9 & 0,93 \\
\hline
\end{tabular}


de massas de aproximadamente de $12 \%$ para as amostras. As curvas de DTA, em geral apresentaram uma deflexão acentuada iniciando em torno de $40{ }^{\circ} \mathrm{C}$ e se estendendo até $200{ }^{\circ} \mathrm{C}$ típica de volatilização de água de hidratação ou adsorvida na superfície do material, relativo a umidade das amostras. O pico endotérmico em $509{ }^{\circ} \mathrm{C}$, observado em ambas amostras, foi associado à reação de desidroxilação do mineral caulinita levando a conversão da metacaulinita [27], de acordo com a Reação 1.

$$
\mathrm{Al}_{2} \mathrm{Si}_{2} \mathrm{O}_{5}(\mathrm{OH})_{4}-\stackrel{>509^{\circ} \mathrm{C}}{\longrightarrow} \mathrm{Al}_{2} \mathrm{Si}_{2} \mathrm{O}_{7}+2 \mathrm{H}_{2} \mathrm{O} \text { reação } 1
$$

Também foi observado que a partir de $600{ }^{\circ} \mathrm{C}$ a estabilidade térmica é garantida para o tratamento térmico (calcinação) nas duas amostras visando a obtenção da metacaulinita como precursor da hidroxisodalita.

Com base na perda de água de desidratação da curva TGA foi estimada a quantidade desse mineral presente em cada amostra, assim a A01-a apresentou teor de $71 \%$ e A14-a de 69\% de abundância.

A Fig. 3 mostra os espectros de infravermelho, em detalhes das faixas 4000 a $2900 \mathrm{~cm}^{-1}$ e 1800 a $400 \mathrm{~cm}^{-1}$, das amostras A01-a (fração fina in natura), A01-b (fração fina calcinada a $600{ }^{\circ} \mathrm{C}$ ) e A01-c (produto da síntese da fração fina). Os mesmos padrões espectrais foram observados para a amostra A14.

Observa-se que o espectro da amostra A01-a apresenta

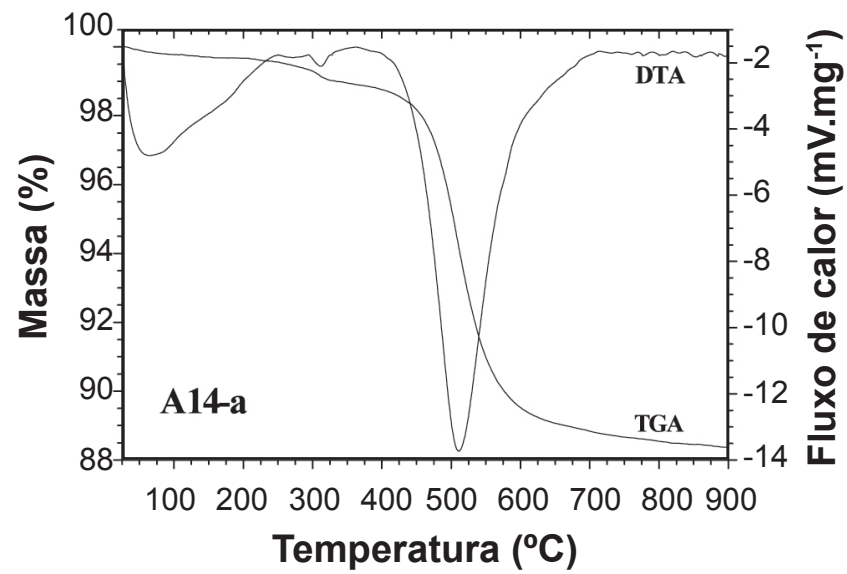

Figura 2: Curvas TGA e DTA da amostra A14-a in natura registrada entre 25 e $900{ }^{\circ} \mathrm{C}$.

[Figure 2: TGA and DTA curves of sample A14-a in natura recorded between 25 and $900^{\circ} \mathrm{C}$.]

bandas relativas às hidroxilas $(\mathrm{O}-\mathrm{H})$ externas em $3695 \mathrm{e}$ $3650 \mathrm{~cm}^{-1} \mathrm{e}$ hidroxilas internas $3619 \mathrm{~cm}^{-1}$, típico do mineral caulinita [28,29]. Já o espectro da amostra A01-b confirmou a conversão total da caulinta em metacaulinita, uma vez que as bandas características da caulinita foram suprimidas e em seu lugar surgiu uma banda intensa, larga e assimétrica em torno de $1063 \mathrm{~cm}^{-1}$ típica da metacaulinita, de acordo com os resultados observados por Breck [4], Akolekar e colaboradores [30] e Rocha e colaboradores [31]. O processo térmico a $600{ }^{\circ} \mathrm{C}$ leva ao colapso da estrutura da caulinita com a formação da metacaulinita, como matéria-prima mais

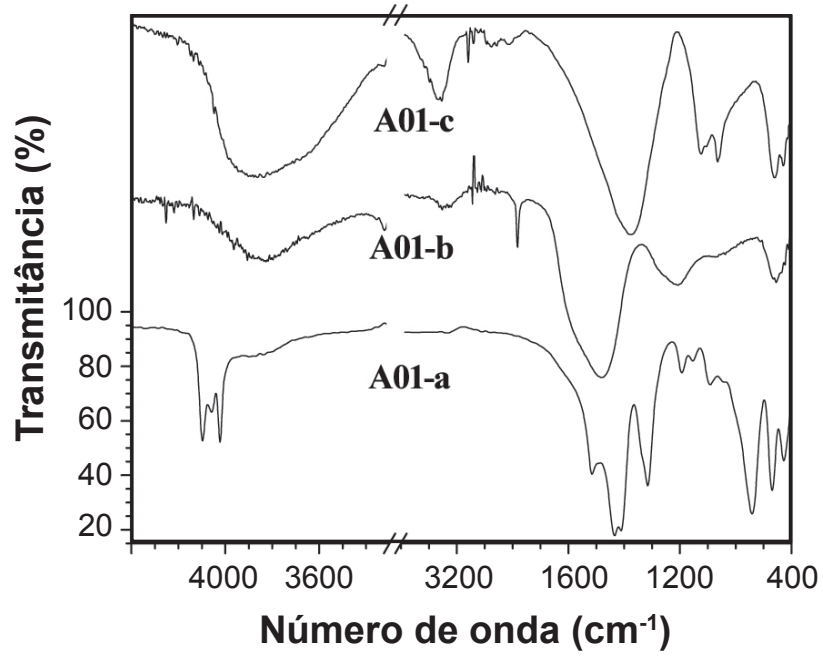

Figura 3: Espectros de infravermelho das amostras A01-a (fração fina in natura), A01-b (fração fina calcinada a $600{ }^{\circ} \mathrm{C}$ ) e A01-c (produto da síntese da fração fina tratada termicamente).

[Figure 3: Infrared spectra of sample A01-a (fraction in natura), A01-b (fine fraction calcined at $600{ }^{\circ} \mathrm{C}$ ) and A01-c (product of synthesis of heat-treated fine fraction).]

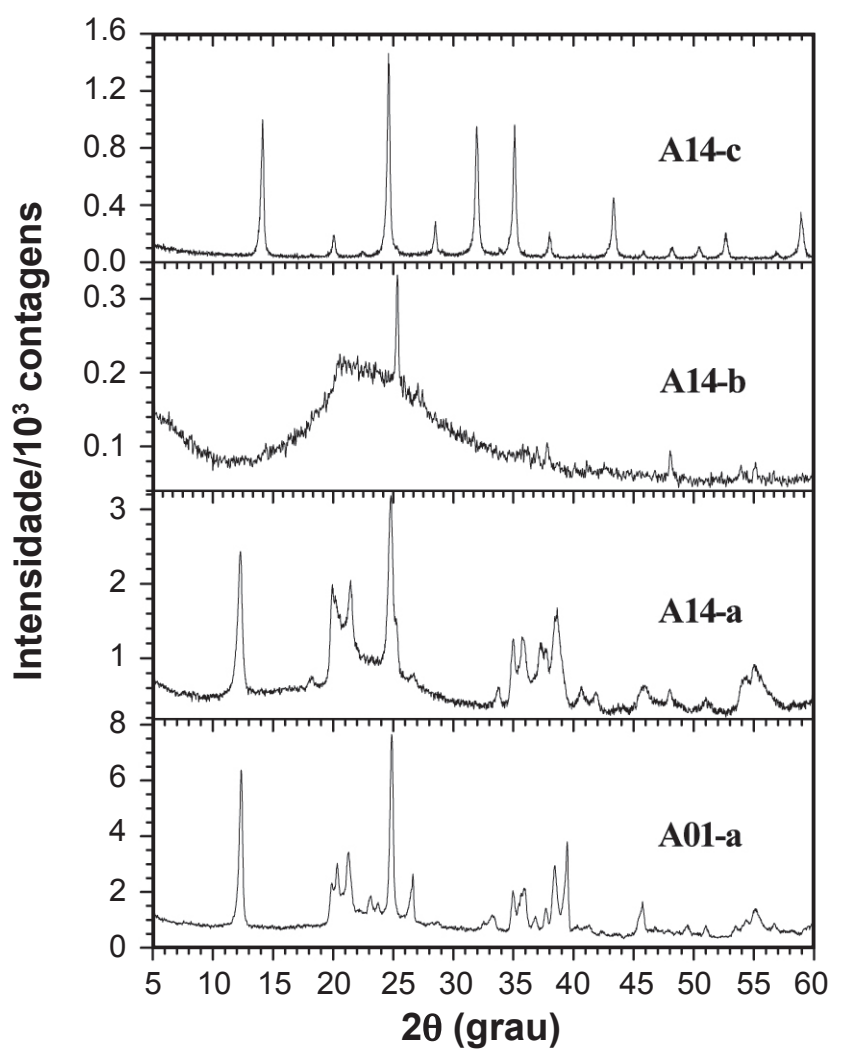

Figura 4: Difratogramas de raios X das amostras A01-a e A14-a (fração fina in natura), A14-b (fração fina calcinada a $600{ }^{\circ} \mathrm{C}$ ) e A14-c (produto da síntese da fração fina tratada termicamente).

[Figure 4: X-ray diffraction patterns of samples A01-a and A14-a (fraction in natura), A14-b (fine fraction calcined at $600{ }^{\circ} \mathrm{C}$ ) and A14-c (product of synthesis of heat-treated fine fraction).]

adequada para a síntese de zeólitas conforme registros nas literaturas. Esses resultados estão em consonância com os observados pelas análises térmicas. 
No espectro da amostra A01-c as bandas observadas em 3477 e $1660 \mathrm{~cm}^{-1}$ são atribuídas às águas zeolíticas. A presença de material zeolítico foi confirmada pela presença das bandas em 1031 e $990 \mathrm{~cm}^{-1}$. Essas bandas estão em torno de $1000 \mathrm{~cm}^{-1}$, que por sua vez é uma banda característica da ligação Si-O-Al do tetraedro $\mathrm{TO}_{4}$. Mas, por outro lado, foram observadas bandas em 672 e $608 \mathrm{~cm}^{-1}$ característica de hidroxisodalita ao invés da zeólita $\mathrm{A}$, de acordo com o trabalho de Maia e colaboradores [6].

A hidroxisodalita é cristalizada em condições hidrotermais, quando se adiciona ao caulim uma solução de hidróxido de sódio. Também pode ser formada a partir de metacaulinita, utilizando essa mesma base por longo período de tempo. Neste caso, primeiramente é formada zeólita A e logo após a hidroxisodalita [6].

Os difratogramas de raios $\mathrm{X}$ das amostras da A01-a e A14-a (fração fina in natura), A14-b (fração fina calcinada a $600{ }^{\circ} \mathrm{C}$ ) e A14-c (produto da síntese da fração fina calcinada) são apresentados na Fig. 4. As amostras A01-b e -A01-c (não mostradas) apresentaram perfis gráficos similares quando comparadas com as respectivas amostras A14-b e A14-c.

Verificou-se que as amostras A01-a e A14-a (Fig. 4) são constituídas essencialmente pela caulinita com a presença das duas principais reflexões em 12,2 \%/2 $(\sim 0,715 \mathrm{~nm}$, plano 001) e $25,1 \% / 2 \theta(\sim 0,356 \mathrm{~nm}$, plano 002). Também foram observadas reflexões incipientes de anatásio $\left(\mathrm{TiO}_{2}\right)$ e quartzo $\left(\mathrm{SiO}_{2}\right)$, em 25,2 \% $/ 2 \theta(\sim 0,352 \mathrm{~nm}$, plano 110) e $26,7^{\circ} / 2 \theta(\sim 0,356 \mathrm{~nm}$, plano 101$)$, respectivamente, quando comparadas com as principais reflexões da caulinita. Estes minerais, geralmente estão presentes nos solos da região amazônica, conforme mostrado na análise química. A análise do difratograma da amostra A14-b, resultante do tratamento térmico, revelou a ocorrência de material amorfo característico da metacaulinita [32], conforme observado também pelas técnicas termogravimétrica e infravermelho. Foi mencionado que a metacaulinita é um material muito mais reativo do que a caulinita para a síntese de zeólitas [30]. A amostra A14-c revelou-se ser muito semelhante com o padrão de difração de raios $\mathrm{X}$ da zeólita hidroxisodalita cristalina disponível na base de dados da IZA-SC [26], onde apresenta reflexões estreitas e bem delineadas, mais de baixas intensidades. Para os refinamentos foram utilizadas inicialmente as coordenadas atômicas para os átomos da estrutura, os parâmetros de rede e o grupo espacial $\mathrm{P} \overline{4} 3 \mathrm{n}$ da estrutura cúbica [26]. Para ambos os testes padrões, o perfil da linha de base (background) foi ajustado com uma função polinomial com 6 coeficientes. O perfil das reflexões da difração foi modelado usando uma função Thompson-CoxHastings pseudo-Voigt (Axial divergence asymmetry) e a largura à meia altura da reflexão com a fórmula de Caglioti, $F W H M^{2}=U \operatorname{tg}^{2} \theta+V \operatorname{tg} \theta+W$ [34]. A origem zero para escala de varredura $2 \theta$, forma e assimetria das reflexões, as ocupações atômicas e os parâmetros térmicos isotrópicos também foram seqüencialmente refinados. Foi adotado o menor valor para o índice $S$ como critério de um bom ajuste, onde $S=R_{w p} / R_{\text {exp }}$, que visa atingir os menores valores dos fatores de confianças $R_{w p}$ e $R_{\text {exp }}$.

Nas Tabelas II e III são mostrados os resultados do refinamento estrutural Rietveld das amostras A01-c e A14-c. O melhor ajuste é indicado pelo valor do índice $S$, onde se obtiveram 1,282 e 1,252 para A01-c e A14-c, respectivamente.

As Figs. 5 e 6 mostram os perfis dos dados experimentais e calculados, ajustados com a melhor descrição gráfica das intensidades da fase cristalina pura da hidroxisodalita indicado pela diferença dos dados experimental e calculado, resultante do refinamento para as respectivas amostras A01-c e A14-c.

Os parâmetros de rede refinados são (i) $a=0,88468$ (3) nm para A01-c e (ii) $a=0,88594(1) \mathrm{nm}$ para A14-c. Esses valores são muito próximos quando comparados com o valor da literatura [34] - ver Tabela II - onde sugere que os tetraedros $\mathrm{TO}_{4}(\mathrm{~T}=\mathrm{Al}$ e $\mathrm{Si})$ da hidroxisodalita ligados entre si estão aparentemente bem ordenados. $\mathrm{O}$ ajuste final das ocupações atômicas relativas para o cálculo da fórmula mínima não afetou os coeficientes estequiométricos, reproduzindo assim, a mesma fórmula inicialmente inserida para o ajuste, $\mathrm{Na}_{6}\left[\mathrm{Si}_{6} \mathrm{Al}_{6} \mathrm{O}_{4}\right]_{6} \cdot 8 \mathrm{H}_{2} \mathrm{O}$ (Tabela III). Com base na posição $2 \theta_{h k l}$ e na largura à meia altura da reflexão ( $\beta=F W H M)$ correspondente de cada reflexão calculada foi estimado o tamanho médio das partículas $(d)$ empregandose a fórmula de Scherrer [35]: $\mathrm{d}_{\mathrm{hkl}}=k . \lambda /\left(\beta \cdot \cos \theta_{\mathrm{hkl}}\right)$, na qual $k$ é a constante de proporcionalidade que depende da forma das partículas $(0,9)$ e $\lambda$ é comprimento de onda da radiação do $\mathrm{Cu}(K \alpha=0,154184 \mathrm{~nm})$. A área específica $S$ (expressa $\mathrm{em} \mathrm{m}^{2} \cdot \mathrm{g}^{-1}$ ) foi calculada através da equação [36]: $\mathrm{S}=6 \times 10^{3} / \rho . d$, na qual $\rho$ é a densidade da hidroxisodalita em $\mathrm{g} . \mathrm{cm}^{-3}$ e $d$ é tamanho médio das partículas expresso em nm. Esses parâmetros são mostrados na Tabela II, em que coincidentemente os valores de tamanhos médios

Tabela II - Resultados obtidos do refinamento estrutural Rietveld, tamanho médio das partículas $(d)$ e área superficial $(S)$ calculadas para as amostras A01-c e A14-c.

[Table II - Results obtained from the Rietveld structural refinement, average particle size (d) and surface area (S) calculated for the samples A01-A14-c.]

\begin{tabular}{cccccccc}
\hline Amostra & $S$ & $R_{w p}$ & $R_{\text {exp }}$ & $a / \mathrm{nm}$ & $V_{c} / \mathrm{nm}^{3}$ & $d / \mathrm{nm}$ & $S / \mathrm{m}^{2} \mathrm{~g}^{-1}$ \\
\hline$[33]$ & - & - & - & 0,88480 & 0,69268 & - & - \\
A01-c & 1,282 & 19,6 & 15,3 & $0,88468(3)$ & $0,69306(4)$ & 57 & 44 \\
A14-c & 1,252 & 13,4 & 10,8 & $0,88594(1)$ & $0,69537(3)$ & 57 & 41 \\
\hline
\end{tabular}


Tabela III - Coordenadas estruturais $(x, y, z)$, fator ocupação (f.o.), parâmetro térmico isotrópico $\left(B_{i s o}\right)$ obtidos no refinamento de Rietveld para o modelo estrutural da hidroxisodalita $\mathrm{Na}_{6}\left[\mathrm{Si}_{6} \mathrm{Al}_{6} \mathrm{O}_{4}\right]_{6} .8 \mathrm{H}_{2} \mathrm{O}$.

[Table III - Structural coordinates $(x, y, z)$, occupancy factor (f.o.), isotropic thermal parameters (Biso) obtained from Rietveld refinement for the model structural Hydroxysodalite $\left.\mathrm{Na}_{6}\left[\mathrm{Si}_{6} \mathrm{Al}_{6} \mathrm{O}_{4}\right]_{6} .8 \mathrm{H}_{2} \mathrm{O}.\right]$

\begin{tabular}{|c|c|c|c|c|c|c|}
\hline Átomo & Íon & $x$ & $y$ & $z$ & f.o. & $\boldsymbol{B}_{\text {iso }}$ \\
\hline \multicolumn{7}{|c|}{ Amostra A01-c } \\
\hline $\mathrm{Na}$ & $\mathrm{Na}^{+}$ & 0,15143 & 0,15413 & 0,15413 & 0,250 & 3,92741 \\
\hline Sil & $\mathrm{Si}^{4+}$ & $1 / 4$ & 0 & $1 / 2$ & 0,250 & 0,58573 \\
\hline Al1 & $\mathrm{Al}^{3+}$ & $1 / 4$ & $1 / 2$ & 0 & 0,250 & 0,64936 \\
\hline $\mathrm{O} 1$ & $\mathrm{O}^{2-}$ & 0,13654 & 0,43737 & 0,14833 & 1,000 & 0,74874 \\
\hline $\mathrm{O} 2\left(\mathrm{H}_{2} \mathrm{O}\right)$ & $\mathrm{O}^{2-}$ & 0,37318 & 0,37318 & 0,37318 & 0,333 & 5,02341 \\
\hline \multicolumn{7}{|c|}{ Amostra A14-c } \\
\hline $\mathrm{Na}$ & $\mathrm{Na}^{+}$ & 0,16169 & 0,16169 & 0,16169 & 0,250 & 5,53378 \\
\hline Sil & $\mathrm{Si}^{4+}$ & $1 / 4$ & 0 & $1 / 2$ & 0,250 & 0,88298 \\
\hline Al1 & $\mathrm{Al}^{3+}$ & $1 / 4$ & $1 / 2$ & 0 & 0,250 & 0,54890 \\
\hline $\mathrm{O} 1$ & $\mathrm{O}^{2-}$ & 0,13839 & 0,43877 & 0,14816 & 1,000 & 1,52660 \\
\hline $\mathrm{O} 2\left(\mathrm{H}_{2} \mathrm{O}\right)$ & $\mathrm{O}^{2-}$ & 0,37801 & 0,37801 & 0,37801 & 0,333 & 11,64096 \\
\hline
\end{tabular}

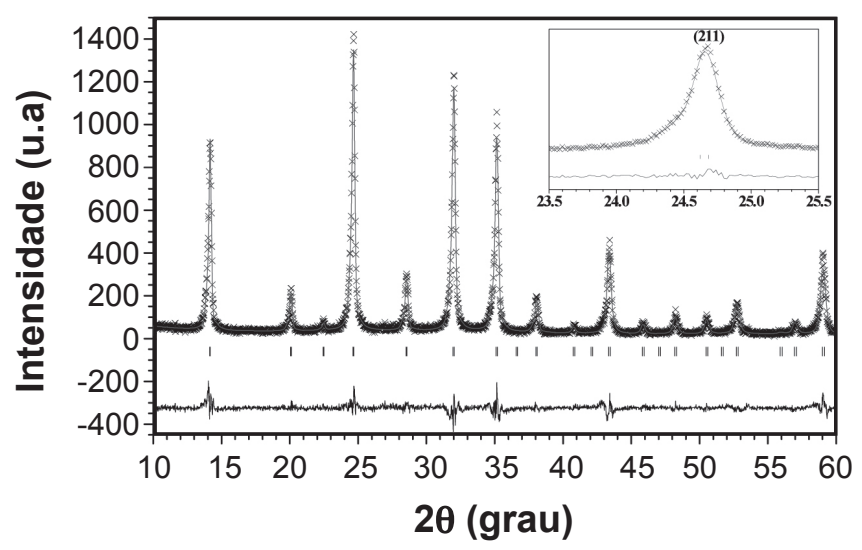

Figura 5: Padrão de DRX refinado pelo método de Rietveld para as amostras A01-c. Padrão experimental (cruzes) e calculado (linha contínua), curva da diferença (linha inferior) e reflexões Bragg (traço vertical) usando o grupo espacial $\mathrm{P} \overline{4} 3 \mathrm{n}$.

[Figure 5: XRD pattern with Rietveld method refinement for samples A01-c. Patterns observed (crosses) and calculated (solid line), the difference curve (bottom line) and Bragg reflections

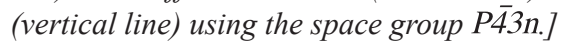

de partícula são iguais para as duas amostras, mas a área superficial da A01-c se mostrou maior em torno de $7 \%$ quando comparados com os valores da A01-c.

\section{CONCLUSÕES}

A fração fina das amostras in natura de solo, A01-a e A14-a, é constituída basicamente de caulinita, com abundância superior a $2 / 3$ confirmada pela termogravimetria e corroborada pela análise química. $\mathrm{O}$ tratamento térmico a $600^{\circ} \mathrm{C}$ foi suficiente para garantir a conversão total de caulinita em metacaulinita nas amostras, tornando-se assim uma

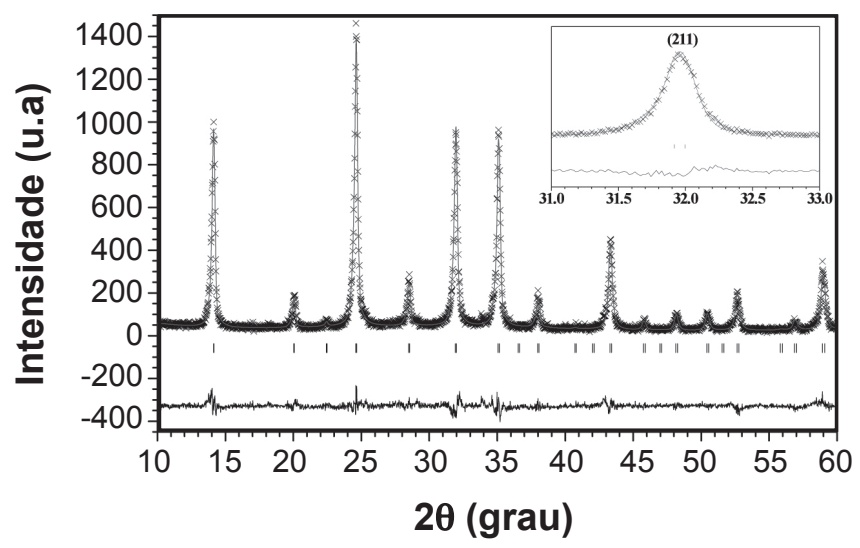

Figura 6: Padrão de DRX refinado pelo método de Rietveld para as amostras A14-c. Padrão experimental (cruzes) e calculado (linha contínua), curva da diferença (linha inferior) e reflexões Bragg (traço vertical) usando o grupo espacial $\mathrm{P} \overline{4} 3 \mathrm{n}$.

[Figure 6: XRD pattern with Rietveld method refinement for samples A14-c. Patterns observed (crosses) and calculated (solid line), the difference curve (bottom line) and Bragg reflections (vertical line) using the space group PÄ3n.]

excelente matéria-prima para produção da hidroxisodalita. As melhores condições para a síntese da hidroxisodalita virtualmente cristalina foram a temperatura de $110{ }^{\circ} \mathrm{C}$ e o tempo de $24 \mathrm{~h}$, que do ponto de vista termodinâmico a zeolita A é uma fase metaestável e tende a se transformar em hidroxisodalita com o tempo [6]. Portanto, a síntese da zeolita A com amostra desse solo (fração fina) pode ser obtida reduzindo-se gradualmente o tempo com mesma temperatura. O refinamento estrutural Rietveld dos dados de DRX para as duas amostras apresentaram parâmetros de rede muito próximos do citado na literatura para hidroxisodalita, o que sugere se tratar de material bastante cristalino. E com 
base nos dados ajustados foi estimado o tamanho médio dos cristalitos e a área superficial.

\section{AGRADECIMENTOS}

Ao Conselho Nacional de Desenvolvimento Científico e Tecnológico (CNPq) pelo apoio financeiro, ao Laboratório de Pesquisas e Ensaios de Combustíveis (LAPEC/UFAM) na obtenção das curvas de termoanálises e ao Laboratório de Difração de raios X do Departamento de Geociências (DEGEO/UFAM) pela obtenção dos difratogramas de raios $\mathrm{X}$.

\section{REFERÊNCIAS}

[1] R. T. Pabalan, F.P. Bertetti, Mineralogical Soc. America 45 (2001) 453.

[2] A. A. B. Maia, R. S. Angélica, R. F. Neves, Cerâmica 54, 331 (2008) 345.

[3] A. B. Luz, Zeólitas: propriedades e usos industriais, CETEM/CNPq, Rio de Janeiro: CETEM/CNPq (1995).

[4] D. W. Breck, Zeolite Molecular Sieves, $2^{\text {nd }}$ John Wiley \& Sons, New York, EUA (1984) 771.

[5] P. Giannetto, Síntese de Zeolitas, Editorial Inovación Tecnológica, Caracas, Venezuela (1990).

[6] A. A. B. Maia, E. Saldanha, R. S. Angélica, C. A. G. Souza, R. F. Neves, Cerâmica 53, 327 (2007) 319.

[7] M. J. Weller, Chem. Soc, Dalton Trans. (2000) 4227.

[8] R. Barrer, J. Cole, J. Chem. Soc. (A) (1970) 1516.

[9] S. Khajavi, F. Kapteijn, J. C. Jansen, J. Membrane Sci. 299 (2007) 63.

[10] F. Ocanto, C. Linares, C. N. Urbina, R. Álvarez, Acta Microscópica 14, 1 e 2 (2005) 15.

[11] Y. Jianfeng, W. Huanting, K. R. Ratinac, S. P. Ringer, Chem. Mater. 18 (2006) 1394.

[12] X. Xiaochun, B. Yun, S. Chunshan, Y. Weishen, L. Jie, L. Liwu, Microporous Mesoporous Mater. 75 (2004) 173.

[13] H. M. Rietveld, J. Applied Crystallography 2 (1969) 65. [14] L. B. McCusker, R. B. Von Dreele, D. E. Cox, D. Loüer, P. Scardi, J. Applied Crystallography 32 (1999) 36.

[15] R. D. Bonetto, P. E. Zalba, M. S. Conconi, M. Manassero, Revista Geológica do Chile 30, 1 (2003) 103.

[16] D. L. Bish, S. J. Howard, J. Appl. Crystallography 21 (1997) 86.

[17] J. Rodríguez-Carvajal, Satellite Meeting on Powder Diffraction of the XV Congress of the IUCr, Toulouse,
França (1990) 127.

[18] R. A. Young, The Rietveld Method, Oxford Univ. Press, London (1993).

[19] J. Bergmann, R. Kleeberg, T. Taut, A. Haase, Advance in X-ray Analysis 40 (1997) 112.

[20] L. A. Gobbo, Aplicação da difração de raios $X e$ método de Rietveld no estudo de cimento Portland, Tese Dr., Universidade de S. Paulo, SP (2009).

[21] M. V. Caputo, R. Rodrigues, D. N. N. Vasconcelos, Litoestratrigrafia da bacia do rio Amazonas, In: C. Schobbenhaus, D. A. Campos, G. R. Derzi, H. E. Asmus, Ed. Geologia do Brasil, Belém: DNPM (Relatório Técnico Interno 691-A, Petrobrás) (1972) 92 p.

[22] R. F. Daemon, C. J. A. Contreiras, Anais XXV Cong. Bras. Geol. S. Paulo, SP 3 (1971) 79.

[23] M. Murat, A. Amokrane, J. P. Bastide, L. Montanaro, Clay Minerals 27, 1 (1992) 119.

[24] Fullprof - www.llb.cea.fr/fullweb/winplotr/winplotr. htlm, version december/2008 (2008).

[25] J. Rodriguez-Carvajal, Short Reference Guide of the Program Fullprof, www.llb.cea.fr/fullweb/winplotr/ winplotr.htlm (2005).

[26] Structure Commission of the International Zeolite Association (SC-IZA) - http://izasc.ethz.ch/fmi/xs1/IZA-SC/ xrd.xsl (2010).

[27] M. Gal, J. Thermal Analysis 37 (1991) 1621.

[28] C. F. Gomes, Argilas. O que são e para que servem?, Fundação Calouste Gulbenkian, Lisboa, Portugal (1986).

[29] J. D. Russel, A. R. Fraser, Clay Mineralogy: Spectroscopic and Chemical Determinative Methods, Chapman and Hall, London, UK (1994).

[30] D. Akolekar, A. Chaffee, R. F. Howe, Zeolites 19, 56 (1997) 359.

[31] J. Rocha, J. Klinowski, J. M. Adams, J. Chem. Soc., Faraday Trans. 87, 18 (1991) 3091.

[32] J. Felsche, S. Luger, C. H. Baerlocher, Zeolites 6, 5 (1986) 367.

[33] E. P. Moraes, N. R. C. F. Machado, S. B. C. Pergher, Acta Scientiarium Technol. 25, 1 (2003) 63.

[34] G. Caglioti, A. Paoletti, F. P. Ricci, Nucl. Instr. 3 (1958) 223.

[35] H. P. Klüg, L. E. Alexander, X-ray Diffraction Procedures for Polycrystalline and Amorphous Materials, John Wiley \& Sons, N. York, EUA (1974) 966.

[36] K. A. Starz, E. Auer, T. Lehmann, R. Zuber, J. Power Sources 84 (1999) 167.

(Rec. 14/04/2010, Ac. 05/08/2010) 